\title{
Electronic spectral and photophysical properties of some $p$-phenylenevinylene oligomers in solution and thin films
}

\author{
J. Seixas de Melo ${ }^{a, *}$, J. Pina ${ }^{a}$, H.D. Burrows ${ }^{a}$, R.E. Di Paolo ${ }^{b}$, A.L. Maçanita ${ }^{b}$ \\ ${ }^{a}$ Departamento de Química da Universidade de Coimbra, Rua Larga, 3004-535 Coimbra, Portugal \\ b Instituto Superior Técnico (IST), Departamento de Química, Lisboa, Portugal
}

Received 27 May 2006; accepted 15 September 2006

Available online 20 September 2006

\begin{abstract}
A comprehensive photophysical and spectroscopic study of a new class of $p$-phenylenevinylene oligomers (PPV-trimers) possessing different alkyl and alkyloxy sidechain substituents and different end groups (aldehyde, $\mathrm{C}=\mathrm{C}$, phenylene and anthracene units) was undertaken in solution at room temperature $(293 \mathrm{~K})$, low temperature $(77 \mathrm{~K})$ and in thin films. The study comprises absorption, emission and triplet-triplet absorption spectra, together with quantitative measurements of quantum yields (fluorescence, intersystem crossing, internal conversion and singlet oxygen formation) and lifetimes. The data allow the determination of rate constants for all decay processes. From these, several conclusions could be drawn. Changing from alkyl to alkyloxy substituents does not change fluorescence and internal conversion yields but decreases the (already small) intersystem crossing yield. The introduction of anthracene at the terminal ends of the PPV-trimers leads to the lowest fluorescence yield reported in this study. Of particular importance is the fact that the fluorescence quantum yields in films are of the same order of magnitude as those in solution, which suggests the potential for use of these oligomers for light-emitting device applications. With one of the alkyloxy derivatives, a more detailed study of the early part of the fluorescence decay was made, and it was found that upon excitation a fast conformational relaxation process of the initially excited oligomer occurs, leading to a more planar conjugation segment.
\end{abstract}

(c) 2006 Elsevier B.V. All rights reserved.

Keywords: Photophysics; Conjugated polymers and oligomers, PPV; Singlet and triplet state; Fluorescence; Quantum yields; Rate constants; Phenylenevinylene

\section{Introduction}

In the last two decades, conjugated organic polymers have attracted interest for various important technological applications, especially those focused on their conducting, electroluminescent and photoluminescent properties and their potential application in electronic and electro-optic devices, in particular as light emitting diodes (LED) [1]. The first of these to be used in LEDs, and still one of the most popular families involves $p$-phenylenevinylene (PPV) derivatives. Numerous experimental and theoretical studies have aimed characterization of electronic states of PPV oligomers and polymers [2-9].

\footnotetext{
* Corresponding author. Tel.: +351 239 854464; fax: +351239827703.

E-mail address: sseixas@ci.uc.pt (J. Seixas de Melo).
}

The detailed understanding of these polymers is a difficult task, involving complex intra- and inter chain dynamics [10] and interactions [11], degradation [12], poor solubility, etc., which makes the study of the corresponding oligomers extremely useful for comparison purposes. The availability of soluble PPV oligomers makes it possible to study their intrinsic molecular properties, and extrapolate to the behaviour of the related polymer $[5,7,13,14]$. Moreover, the study of these compounds in dilute solutions allows their characterization in the absence of significant effects due to interchain interactions and under conditions where the time between collisions of two oligomers is longer than the lifetimes of the excited states under investigation. However, although these studies are valuable, the characterization of these polymers in thin films must be one of the main goals, since for practical application these 
conjugated organic polymers are almost always found in solid state devices, with the production of thin film light emitting devices (LED) being one of their major applications. For such applications, one important drawback of these compounds is related to the formation of excited triplet states. These are expected to be the predominant species formed by charge recombination in electroluminescent devices (75\% from spin statistics) [4] and because singlet oxygen formation (via interaction with oxygen) is observed following photolysis of aerated solutions of conjugated polymers [15], it may have important implications in a device working and consequently lead to degradation of devices based on conjugated polymers [12]. Triplet states of conjugated polymers have, in the solid state, lifetimes of the order of a few milliseconds, which again make them very susceptible to degradation induced by light. In addition, they also show high extinction coefficients for optical transition to higher energy states in the triplet manifold $[4,16]$, which can affect their potential for use in all organic laser systems. Moreover, since triplet states of conjugated polymers, in the absence of appropriate metal complex triplet emitters, are generally devoid of phosphorescence, radiationless processes will be one of the major energy loss mechanisms in LED devices.

A complete knowledge on the formation and decay pathways of all processes is therefore mandatory for a full understanding of the electroluminescence behaviour of conjugated organic polymers. Following this strategy we have made a systematic and complete photophysical study on six monodisperse substituted trimer derivatives of PPV.

\section{Experimental}

\subsection{Materials}

The compounds were a kind gift from Professor ThornCsányi (University of Hamburg). All compounds were synthesized as elsewhere described $[7,17,18]$.

Solvents were of spectroscopic or equivalent grade. Methylcyclohexane $(\mathrm{MCH})$ was purified by the procedure described elsewhere [19].

\subsection{Spectroscopic measurements}

The absorption and emission experiments were made in $\mathrm{MCH}$, with trimer concentrations in the range $1 \times 10^{-5}$ $10^{-6} \mathrm{M}$. These were deoxygenated by bubbling with Ar.

Absorption and fluorescence spectra were recorded on Shimadzu UV-2100 and Horiba-Jobin-Ivon SPEX Fluorog 3-22 spectrometers, respectively. The fluorescence spectra were corrected for the wavelength response of the system.

The fluorescence quantum yields were measured using quinquethiophene as standard, $\phi_{\mathrm{F}}=0.33$ [20]. The fluorescence quantum yields at low temperature were obtained by running under the same experimental conditions the spectra done at $293 \mathrm{~K}$. The $\phi_{\mathrm{F}}$ value was then obtained by assuming $V_{77 \mathrm{~K}} / V_{293 \mathrm{~K}}=0.8$ [21], i.e. a $20 \%$ contraction of the solvent on going from $293 \mathrm{~K}$ to $77 \mathrm{~K}$.

The experimental setup used to obtain triplet spectra and triplet yields consists of an Applied Photophysics laser flash photolysis apparatus pumped by a Nd:YAG laser (Spectra Physics). The detection system is at right angles to the excitation beam and a pulsed $150 \mathrm{~W}$ Xe lamp is used to analyze the transient absorption. The signal obtained is fed into a HP digital analyzer and transferred to an IBM RISC computer where using the appropriate software (Applied Photophysics) the OD at different wavelengths and different delays after flash are collected. The transient spectra were obtained, following excitation at $355 \mathrm{~nm}$ with an unfocused laser beam, by monitoring the optical density change at intervals of 5-10 nm over the $250-700 \mathrm{~nm}$ range and averaging at least 10 decays at each wavelength. Firstorder kinetics were observed in all cases for the decay of the lowest triplet state. Special care was taken in determining triplet yields to have optically matched dilute solutions (abs $\approx 0.2$ in a $10 \mathrm{~mm}^{2}$ cell) and low laser energy $(\leqslant 2 \mathrm{~mJ})$ to avoid multiphoton and $\mathrm{T}-\mathrm{T}$ annihilation effects.

The triplet molar absorption coefficients were determined by the singlet depletion technique, according to the well-known relationship [22]:

$\varepsilon_{\mathrm{T}}=\frac{\varepsilon_{\mathrm{S}} \times \Delta \mathrm{OD}_{\mathrm{T}}}{\Delta \mathrm{OD}_{\mathrm{S}}}$

where both $\Delta \mathrm{OD}_{\mathrm{S}}$ and $\Delta \mathrm{OD}_{\mathrm{T}}$ are obtained from the triplet-singlet difference transient absorption spectra. The $\phi_{\mathrm{T}}$ values were obtained by comparing the $\Delta \mathrm{OD}$ at $525 \mathrm{~nm}$ of a benzene solution of benzophenone (the standard) and of the compound (optically matched at the laser wavelength) using the equation $[23,24]$ :

$\phi_{\mathrm{T}}^{\mathrm{cp}}=\frac{\varepsilon_{\mathrm{TT}}^{\text {Benzophenone }}}{\varepsilon_{\mathrm{TT}}^{\mathrm{cp}}} \times \frac{\Delta \mathrm{OD}_{\max }^{\mathrm{cp}}}{\Delta \mathrm{OD}_{\max }^{\text {Benzophenone }}} \times \phi_{\mathrm{T}}^{\text {Benzophenone }}$

Fluorescence decays were measured using a home-built TCSPC apparatus with an IBH NanoLED (373 or $460 \mathrm{~nm}$ ) excitation source, Jobin-Ivon excitation and emission monochromators, Philips XP2020Q photomultiplier, and Canberra instruments TAC and MCA. Alternate measurements (1000 c.p.c.) of the pulse profile at the excitation wavelength and the sample emission were performed until $5 \times 10^{4}$ counts at the maximum were reached. When picosecond resolution was needed, the fluorescence decays were obtained in an apparatus described elsewhere [25]. The fluorescence decays were analysed using the modulating functions method of Striker with automatic correction for the photomultiplier "wavelength shift" [26].

Room-temperature singlet oxygen phosphorescence was detected at $1270 \mathrm{~nm}$ using a Hamamatsu R5509-42 photomultiplier, cooled to $193 \mathrm{~K}$ in a liquid nitrogen chamber (Products for Research model PC176TSCE-005), following laser excitation of aerated solutions at $355 \mathrm{~nm}$ 
$(\mathrm{OD} @ 355 \mathrm{~nm}=0.20)$, with an adapted Applied Photophysics flash kinetic spectrometer. The modification of the spectrometer involved the interposition of a Scotch RG665 filter. A 600-line diffraction grating was used instead of the standard spectrometer one to extend spectral response to the infrared. The filter employed is essential to eliminate all the first harmonic contributions from the sensitizer emission in the $500-800 \mathrm{~nm}$ region from the infrared signal. $1 H$-Phenalenone (perinaphthenone) in benzene $\left(\phi_{\Delta}=0.93\right)$ was used as standard [21]. To check the precision of these values singlet oxygen yields were verified at the FRRF (Daresbury laboratories), with a set-up elsewhere described [15].

Thin films from the compounds were obtained with a Desk-Top Precision Spin Coating System, Model P6700 Series from Speedline Technologies. The solid-state thin film from the samples were obtained by deposition of a few drops from a solution of the compounds into a circular sapphire substrate $(10 \mathrm{~mm}$ diameter $)$ followed by spin coating $(2500 \mathrm{rpm})$ in a nitrogen saturated atmosphere $(2 \mathrm{psi})$. The solutions for spin-coating were prepared by adding $2 \mathrm{mg}$ of the samples to $15 \mathrm{mg}$ of Zeonex ${ }^{\circledR}$ in $200 \mu \mathrm{L}$ toluene solution with stirring at $40{ }^{\circ} \mathrm{C}$ for $30 \mathrm{~min}$.

The fluorescence emission spectra of the thin films were obtained with a Horiba-Jobin-Yvon integrating sphere. The solid-state photoluminescence quantum yields in thin films were obtained with this integrating sphere, using the method outlined by de Mello et al. [27] and applied by Palsson and Monkman [28] to polypyridine films using a set-up similar to the used in this work.

\section{Results and discussion}

The structures and acronyms of the compounds studied are depicted in Scheme 1. These consist of $p$-phenylenevinylene trimers (PPV-trimers) substituted in the positions 2 and 5 of each terminal phenylene group possessing different alkyl and alkyloxy sidechain. The substituents were 2,5diheptyl with the polymer backbone propenyl end capped (HPV3P), 2,5-di-2'-methylpropyl-oxy with aldehyde (MPOPV3A) end groups or 2,5-di-2'-ethylhexyl-oxy with propenyl (EHOPV3P), aldehyde (EHOPV3A), propenylbenzene (EHOPV3PB) and propenylanthracene (EHOPV3PA) end groups.

\subsection{Solution spectroscopic behaviour}

\subsubsection{Absorption}

The absorption spectra of the PPV trimers were broad and devoid of vibrational structure (Fig. 1); this is a commonly observed feature with related compounds $[7,17,18]$ and in particular with the correspondent polymers $[15,29]$, and is attributed to the wide range of vibrational and rotational degrees of freedom available in conjugated oligomers and polymers [16]. Substitution of an alkyl (HPV3P) by an alkyloxy (EHOPV3P, MPOPV3A) sidechain red-shifted the absorption maximum of the compound by $\approx 67 \mathrm{~nm}$ for the ethylhexyloxy and $\approx 80 \mathrm{~nm}$ for the methylpropyl-oxy substituents, see Table $1[7,17,18]$. For the PPV-trimers with ethylhexyloxy sidechain (EHOPV3P) substitution of the terminal end groups by the aromatic phenylene (EHOPV3PB) and anthracene (EHOPV3PA) groups red-shifted the absorption maximum

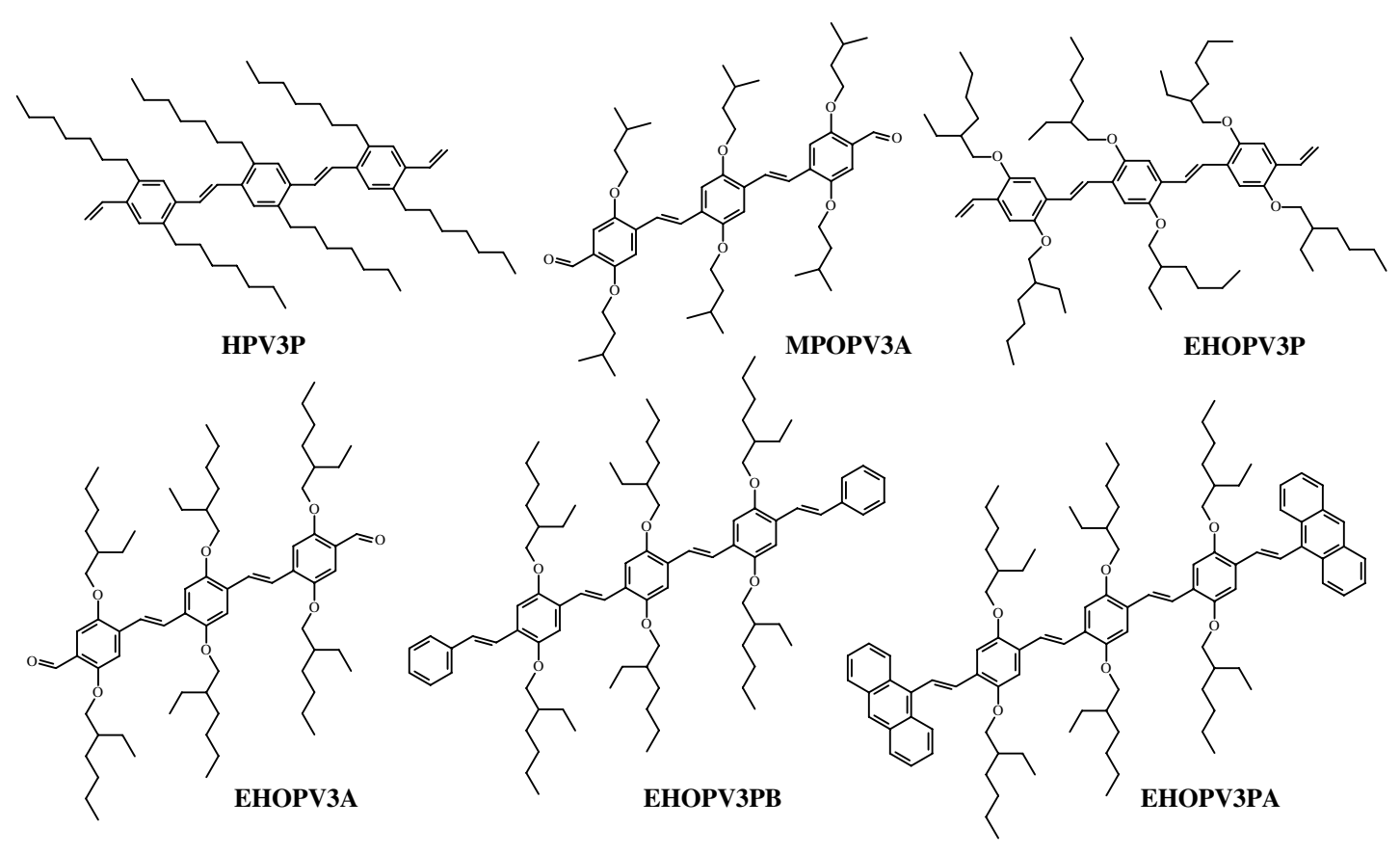

Scheme 1. 

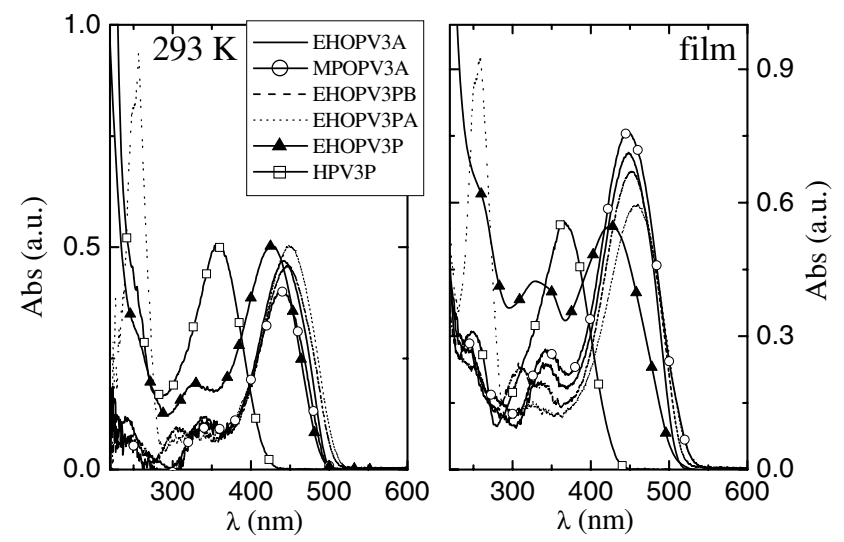

Fig. 1. Absorption spectra of PPV-trimers in methylcyclohexane at room temperature $(293 \mathrm{~K})$ and in the solid-state (film).

by ca. $19 \mathrm{~nm}$ and $23 \mathrm{~nm}$, respectively. This suggests that the $\pi$-conjugation of the PPV skeleton is extended to these terminal groups. With the end-capped anthracene compound (EHOPV3PA) the absorption spectra does not display the long wavelength band, characteristic of anthracene, which suggests efficient energy transfer from anthracene to the $p$-phenylenevinylene (PPV) moiety.

\subsubsection{Fluorescence}

The fluorescence spectra (Fig. 2) and quantum yields in methylcyclohexane (see Table 2) were obtained at room temperature $(293 \mathrm{~K})$ and low temperature $(77 \mathrm{~K})$. In contrast to what was observed in absorption, the emission spectra, in solution at $293 \mathrm{~K}$, show vibrational structure. In solution at $293 \mathrm{~K}$, substitution of alkyl sidechain by alkyloxy red-shifts the fluorescence maximum $\approx 60 \mathrm{~nm}$. For the PPV-trimers with ethylhexyloxy sidechain (EHOPV3P) substitution of the terminal end groups by the aromatic phenylene (EHOPV3PB) and anthracene (EHOPV3PA) groups red-shifts the fluorescence maximum by ca. $23 \mathrm{~nm}$ and $37 \mathrm{~nm}$, respectively. With the compound EHOPV3PA, the fluorescence emission spectrum when obtained with excitation at $255 \mathrm{~nm}$ (corresponding to the $\mathrm{S}_{0} \rightarrow \mathrm{S}_{4}$ anthracene transition [30]) did not display the characteristic emission band of anthracene, supporting

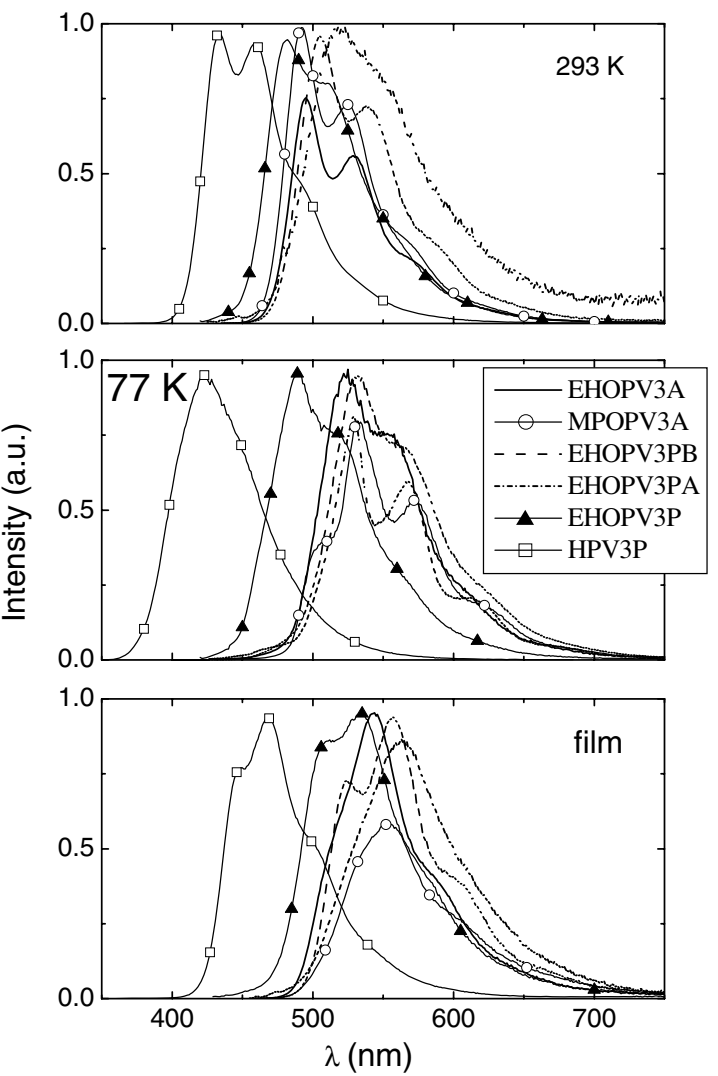

Fig. 2. Fluorescence emission spectra of the studied samples in methylcyclohexane at room temperature $(293 \mathrm{~K})$, low temperature $(77 \mathrm{~K})$ and in thin films.

the idea of efficient energy transfer from the anthracene (terminal groups) to the oligomer backbone.

Upon going to low temperature (LT), the emission spectra, with exception of HPV3P, revealed a red-shift of the band maxima (Fig. 2). With HPV3P a blue shift of the emission band, and loss of vibrational structure, was observed upon going to LT. This phenomena has previously been observed with analogous compounds and was explained in terms of the ground-state geometry to which $\mathrm{S}_{1}$ deactivates with the loss of vibrational structure possibly evidencing the occurrence of self-absorption [7]. The observation of the opposite behaviour to that found for HPV3P

Table 1

Spectral data (absorption, emission wavelength maxima, triplet maxima and extinction - singlet and triplet - coefficients) for the PPV-trimers in methylcyclohexane at room temperature $(293 \mathrm{~K})$, low temperature and in films

\begin{tabular}{|c|c|c|c|c|c|c|c|c|}
\hline Compound & $\begin{array}{l}\lambda_{\max }^{\mathrm{Abs}}(\mathrm{nm})^{\mathrm{b}} \\
\text { Solution } 293 \mathrm{~K}\end{array}$ & $\begin{array}{l}\lambda_{\max }^{\mathrm{Abs}_{1}}(\mathrm{~nm})^{\mathrm{b}} \\
\text { Film }\end{array}$ & $\begin{array}{l}\lambda_{\text {max }}^{\text {Fluo }}(\mathrm{nm})^{\mathrm{b}} \\
\text { Solution } 293 \mathrm{~K}\end{array}$ & $\begin{array}{l}\lambda_{\max }^{\text {Fluo }}(\mathrm{nm})^{\mathrm{b}} \\
\text { Solution } 77 \mathrm{~K}\end{array}$ & $\begin{array}{l}\lambda_{\max }^{\text {Fluo }}(\mathrm{nm})^{\mathrm{b}} \\
\text { Film }\end{array}$ & $\lambda_{\max }^{T_{1} \rightarrow \mathrm{T}_{n}}(\mathrm{~nm})$ & $\varepsilon_{\mathrm{S}}\left(\mathrm{M}^{-1} \mathrm{~cm}^{-1}\right)$ & $\varepsilon_{\mathrm{T}}\left(\mathrm{M}^{-1} \mathrm{~cm}^{-1}\right)$ \\
\hline MPOPV3A & 339,440 & $343, \underline{448}$ & $\underline{492}, 525$ & $\underline{533}, 572$ & 553 & 605 & 42,400 & 81,800 \\
\hline EHOPV3A & $339, \overline{442}$ & $343, \overline{448}$ & $\underline{495}, 529$ & $\overline{523}$ & 543 & 605 & 42,400 & 81,900 \\
\hline EHOPV3PA & $\underline{255}, 450$ & $\underline{258}, 325, \overline{458}$ & $\overline{519}$ & 532 & 563 & - & - & - \\
\hline EHOPV3P $^{\mathrm{a}}$ & 330,427 & 330,427 & 482 & 490 & $510, \underline{534}$ & 560 & 63,000 & 122,940 \\
\hline HPV3P $\mathrm{P}^{\mathrm{a}}$ & 360 & $250, \overline{365}$ & $\underline{434}, 458$ & 426 & 468 & 565 & 52,200 & 195,600 \\
\hline
\end{tabular}

\footnotetext{
${ }^{a}$ Data from Ref. [7].

b The underlined values are the wavelength maxima.
} 
Table 2

Photophysical properties for the PPV-trimers in MCH at room temperature (293 K), low temperature (77 K) and in films

\begin{tabular}{|c|c|c|c|c|c|c|c|c|c|c|c|c|c|c|c|}
\hline Compounds & $\begin{array}{l}\phi_{\mathrm{F}} \\
(293 \mathrm{~K})\end{array}$ & $\begin{array}{l}\phi_{\mathrm{F}} \\
(77 \mathrm{~K})\end{array}$ & $\begin{array}{l}\tau_{\mathrm{F}} \\
(\mathrm{ns})\end{array}$ & $\phi_{\mathrm{T}}$ & $\phi_{\text {IC }}$ & $\begin{array}{l}\tau_{\mathrm{T}} \\
(\mu \mathrm{s})\end{array}$ & $\phi_{\Delta}$ & $\begin{array}{l}k_{\mathrm{F}}\left(\mathrm{ns}^{-1}\right) \\
\text { Solution } \\
293 \mathrm{~K}\end{array}$ & $\begin{array}{l}k_{\mathrm{NR}}\left(\mathrm{ns}^{-1}\right) \\
\text { Solution } \\
293 \mathrm{~K}\end{array}$ & $\begin{array}{l}k_{\text {ISC }}\left(\mathrm{ns}^{-1}\right) \\
\text { Solution } \\
293 \mathrm{~K}\end{array}$ & $\begin{array}{l}k_{\mathrm{IC}}\left(\mathrm{ns}^{-1}\right) \\
\text { Solution } \\
293 \mathrm{~K}\end{array}$ & $\begin{array}{l}\phi_{\mathrm{F}} \\
\text { Film }\end{array}$ & $\begin{array}{l}\tau_{\mathrm{F}} \\
(\mathrm{ns}) \\
\text { Film }\end{array}$ & $\begin{array}{l}k_{\mathrm{F}} \\
\left(\mathrm{ns}^{-1}\right) \\
\text { Film }\end{array}$ & $\begin{array}{l}k_{\mathrm{NR}} \\
\left(\mathrm{ns}^{-1}\right) \\
\text { Film }\end{array}$ \\
\hline MPOPV3A & 0.72 & 0.26 & $1.61^{\mathrm{b}}$ & 0.062 & 0.22 & 52 & 0.064 & 0.447 & 0.174 & 0.039 & 0.137 & 0.67 & 2.35 & 0.285 & 0.14 \\
\hline EHOPV3A & 0.71 & 0.28 & 1.64 & 0.060 & 0.23 & 73 & 0.059 & 0.433 & 0.177 & 0.037 & 0.14 & 0.89 & 1.94 & 0.459 & 0.057 \\
\hline EHOPV3PB & 0.23 & 0.37 & 0.72 & 0.041 & 0.73 & 35 & 0.042 & 0.32 & 1.07 & 0.057 & 1.014 & 0.38 & 0.97 & 0.39 & 0.64 \\
\hline EHOPV3PA & 0.0040 & 0.17 & 1.68 & - & $0.99^{\mathrm{c}}$ & - & $0.002_{6}$ & $0.002_{4}$ & 0.593 & $0.0015^{\mathrm{c}}$ & 0.589 & 0.025 & 2.97 & 0.0084 & 0.33 \\
\hline EHOPV3P ${ }^{\mathrm{a}}$ & 0.61 & 0.44 & 1.72 & 0.025 & 0.365 & 94 & - & 0.355 & 0.226 & 0.015 & 0.212 & 0.69 & 2.34 & 0.295 & 0.132 \\
\hline $\mathrm{HPV} \mathrm{P}^{\mathrm{a}}$ & 0.64 & 0.60 & 1.40 & 0.005 & 0.355 & 3.9 & - & 0.457 & 0.257 & 0.0036 & 0.254 & 0.43 & 1.66 & 0.259 & 0.343 \\
\hline
\end{tabular}

${ }^{a}$ Data from Ref. [7].

${ }^{\mathrm{b}}$ Value obtained with a nanosecond TCSPC instrument. The major component obtained with a picosecond time resolution is $1.48 \mathrm{~ns}$ (see Fig. 4).

${ }^{\mathrm{c}}$ Calculated assuming $\phi_{\mathrm{T}} \approx \phi_{\Delta}$.

with the compounds possessing alkyloxy derivatives may lie in the structural modification induced by the alkyloxy vs. alkyl sidechain, as has previously been observed $[7,17,18]$ and discussed theoretically [31]. The electrondonor characteristics of the alkyloxy group will extend the $\pi$-electrons of the oxygen atoms to the oligomer backbone and as consequence the absorption and emission wavelength maxima are lower in energy. The absence of differences in the shape of the emission spectra upon going from $293 \mathrm{~K}$ to $77 \mathrm{~K}$ also indicates that no significant structural differences have been induced by changing the temperature.

\subsubsection{Triplet state}

Following laser flash photolysis at $355 \mathrm{~nm}$ of degassed solutions of the compounds in $\mathrm{MCH}$, depletion of the ground state absorption between $250 \mathrm{~nm}$ and $480 \mathrm{~nm}$, and formation of a new band in the $400-700 \mathrm{~nm}$ was observed in all cases except for EHOPV3PA (Fig. 3). In this particular case within the experimental conditions used, no triplet signal could be observed which presumably reflects the very low intersystem crossing yield for this particular compound.

As previously observed [7], substitution of alkyl by alkyloxy sidechain in the $p$-phenylenevinylene trimer back-

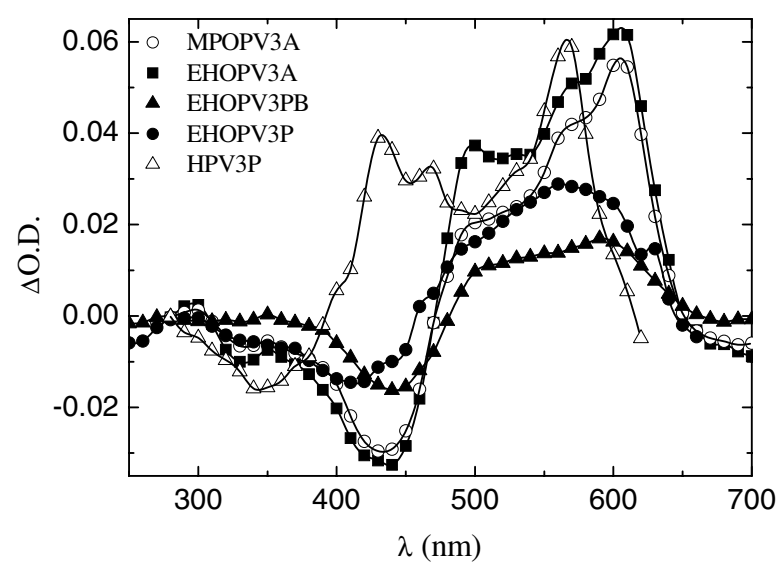

Fig. 3. Triplet-singlet difference spectra for the PPV-trimers in methylcyclohexane at room temperature. bone blue-shifts the transient triplet-triplet absorption spectra maximum. In contrast, with the PPV-trimers with akyloxy sidechain and end-capped with groups possessing an oxygen atom the spectra showed a red-shift in the triplet absorption maximum when compared to EHOPV3P. This suggests that the triplet state with these derivatives is more delocalized than in HPV3 and EHOPV3P, in agreement with the behaviour found in the singlet state. A decrease in the $\mathrm{S}_{1}-\mathrm{S}_{0}$ gap as the conjugation length increases is generally accepted to imply an increased conjugation length delocalizing excitations along the oligomer or polymer backbone [32]. In this particular case the observed pattern should be related with the increase of the electron-donor characteristics of the substituents as the number of oxygen atoms increases. The electron-donor alkyloxy sidechain and terminal end groups will extend the $p-\pi$ electrons of the oxygen atoms to the oligomer backbone and as consequence the HOMO-LUMO energy gap is significantly reduced $[7,18]$. This behaviour is also observed in the triplet state as can be noted by the high delocalization observed in this case as the number of oxygen atoms increases in the terminal and sidechain substituents.

\subsection{Solid-state spectroscopic behaviour}

The absorption and emission spectra of thin films in the solid state (obtained from spin-coated thin film layer) of the PPV-trimers in a Zeonex ${ }^{\circledR}$ matrix are also shown in Figs. 1 and 2. The thin film absorption spectra of these compounds are, when compared with solution data, redshifted $\approx 5-8 \mathrm{~nm}$, with the exception of EHOPV3P whose maxima in solution (RT) and film is $427 \mathrm{~nm}$ (see Table 1). The red-shift of the absorption and emission band upon going from solution to film can be attributed to the loss of conformational freedom, due to the rigid media where the oligomer is immobilized, minimizing twisting vibrations along the PPV-trimer backbone with the consequent lowering of the energy gap between the HOMO and LUMO orbitals, resulting in a red shift of the absorption and fluorescence spectra [18].

For HPV3P, in the solid state matrix, an opposite behaviour was observed to that found at low temperature $(77 \mathrm{~K})$. In the solid state there is a general tendency for 
aggregation to occur, thus forcing the polymer to adopt a more planar geometry in both the ground and excited states. This gives rise to a decrease in the transition energy translated by the marked red-shift observed in the fluorescence emission spectra of HPV3 in thin films.

\subsection{Solution and solid-state photophysical behaviour}

From the laser flash photolysis experiments it was possible to obtain the intersystem crossing singlet-to-triplet quantum yields $\left(\phi_{\mathrm{T}}\right)$ and from room temperature phosphorescence of singlet oxygen sensitization the quantum yields for singlet oxygen formation $\left(\phi_{\Delta}\right)$. Together with the $\phi_{\mathrm{F}}$ values, the overall set of data allows the determination of all the relevant photophysical parameters for the oligomers under study (see Table 2) and thus a consistent discussion based on the all possible deactivation pathways is now possible.

In general it can be seen that the PPV-trimers (MPOPV3A, EHOPV3A, EHOPV3P and HPV3P) which do not have aromatic end-caps present high fluorescence quantum yields (more than $60 \%$ of the quanta are loss via this process). Introduction of the phenyl group (EHOPV3PB) or anthracene (EHOPV3PA) at the endpositions of the PPV-trimers strongly decreases the fluorescence quantum yield $\left(\phi_{\mathrm{F}}\right)$. This is particularly marked in the case of EHOPV3PA since the $\phi_{\mathrm{F}}$ value decreases by three orders of magnitude with the concomitant increase of the internal conversion quantum yield $\left(\phi_{\text {IC }}\right)$. The triplet state quantum yield, albeit lower that for the analogs (MPOPV3A and EHOP3VA), does not seem to change significantly, suggesting that the major change is, in fact, promoted by the increase of the $S_{1} \rightarrow S_{0}$ deactivation channel.

The fluorescence quantum yields at low temperature are in general identical or lower than those obtained at RT. The exceptions occur with the aromatic end-terminated EHOPV3PB and EHOPV3PA, in particular with the latter compound where an increment of two orders of magnitude is observed (Table 2). In the case of EHOPV3P and HPV3P, where virtually no change in the $\phi_{\mathrm{F}}$ value is observed upon going to $77 \mathrm{~K}$ this was attributed to the fact that the $\mathrm{S}_{1}$ state probably has the same geometry as that found at RT [7]. The decrease of the $\phi_{\mathrm{F}}$ values upon lowering the temperature contrasts with the behaviour found for thiophene oligomers where an increase or constancy of this value was observed [20,23]. Thus the marked change (either increase or decrease) in the $\phi_{\mathrm{F}}$ values upon going to LT can be associated with induced conformational changes, which is also in agreement with the marked redshift of the absorption and emission spectra when compared to the RT data.

The replacement of the alkyl (HPV3P) by the alkyloxy (all the other PPV-trimers) sidechain in the PPV skeleton leads to an increase by one order of magnitude of the $\mathrm{S}_{1} \rightarrow \mathrm{T}_{1}$ intersystem crossing yield. A similar effect was previously observed and explained by the increase in the spinorbit coupling due to the presence of the oxygen atom [7].
However, from Table 2 it can be observed that intersystem crossing (ISC) is an inefficient way of deactivation of the excited state of these PPV-trimers as showed by the low values observed for $\phi_{\mathrm{T}}$ (and $\phi_{\Delta}$ ).

From Table 2 it is also notable that, in general, the fluorescence quantum yield in solid state matrices increases relative to liquid solutions (in some cases by more than a factor of two) with the exception of EHOPV3PA and, to a lesser degree, HPV3P. This is in contrast to the behaviour commonly found for $\pi$-conjugated systems which display lower fluorescence quantum yields in the solid state due to the increase of non-radiative decay processes $[33,34]$.

In solution $(293 \mathrm{~K})$ the radiative rate constant $\left(k_{\mathrm{F}}\right)$ is approximately constant with values ranging from $0.32 \mathrm{~ns}^{-1}$ to $0.46 \mathrm{~ns}^{-1}$, with the above mentioned exception of the anthracene end-terminated PPV-trimer. It is also worth noting that the dominant radiationless rate constant is $k_{\mathrm{IC}}$, see Table 1, and that this is even bigger than $k_{\mathrm{F}}$ in the cases of EHOPV3PB and EHOPV3PA, i.e, when aromatic units are capped at the terminals of the PPV-trimers skeleton. The same pattern can be observed in the solid state, although separation of the radiationless rate constants could not be made since $\phi_{\mathrm{T}}$ values in the solid state are not trivial to obtain. However, the comparison of the $k_{\mathrm{NR}}$ rate constants for solid-state matrices and fluid solution media (Table 2) clearly shows a general decrease in the former case. This is an important fact and is in line with the discussed general increase of the fluorescence yields in the solid state.

The pattern of substitution with aromatic units at the terminal positions of the PPV-trimers strongly decreases the efficiency of the radiative channel. This happens with the concomitant increase of the internal conversion deactivation channel. It is likely that the addition of these aromatic units makes a range of vibrational levels available which were not present with the unsubstituted EHOPV3A and EHOPV3P, thus increasing the number of coupling modes between the $S_{1}$ and $S_{0}$ states.

From Table 2 it can also be seen that the singlet oxygen formation yields are low and roughly constant with values lying between 0.042 and 0.064 with the exception of EHOPV3PA where this value is even lower, $\phi_{\Delta} \approx 0.0026$ (see Table 2). This again is associated with ISC not being the favoured pathway for the deactivation of the singlet states of the PPV-Trimers studied. Note also that $\phi_{\Delta}$ values are very close to the quantum yields for triplet formation, providing support for the latter values, and indicating that the efficiency of triplet energy transfer to produce singlet oxygen $\left(\mathrm{S}_{\Delta}=\phi_{\Delta} / \phi_{\mathrm{T}}\right)$ is very close to unity, suggesting very efficient energy transfer from the PPV-Trimer triplet states to ground-state triplet molecular oxygen.

\subsection{Structural relaxation followed by picosecond time- resolved fluorescence}

The conformational behaviour of conjugated polymers has been recently discussed for polyfluorene derivatives 
[10], where it was suggested that upon excitation the planarization of the polymer backbone occurs within the first ps after excitation. Alternative explanations with other conjugated polymers have attributed this relaxation phenomenon to resonance energy transfer (RET) [35], presumably between different polymer segments. These two apparently conflicting explanations seem to question the real nature of the mechanism responsible for the relaxation of these systems.

To provide deeper insight into the relaxation process along the polymer chain, we have studied in more detail the monodisperse MPOPV3A PPV-trimer, see Scheme 1, in methylcyclohexane by time-resolved fluorescence techniques, as a function of both the emission wavelength and the temperature, covering the entire luminescence spectrum. With such trimers, there is no possibility for intrachain energy transfer, and at the low concentrations studied interchain transfer is likely to be insignificant.

In general the fluorescence decay times do not show any significant change along the fluorescence spectrum and can be considered to be independent of the emission wavelength $\left(\lambda_{\mathrm{em}}\right)$ over the whole range studied. However, different relative contributions are observed, which are attested by the change in the pre-exponential factors with the $\lambda_{\mathrm{em}}$. This allows a global analysis of the temporal profiles of fluorescence. In Fig. 4 the fluorescence decay obtained at room temperature and collected at three different $\lambda_{\mathrm{em}}$ exemplifies the general behaviour found. In agreement with the coexistence of more than one conformer, only triple

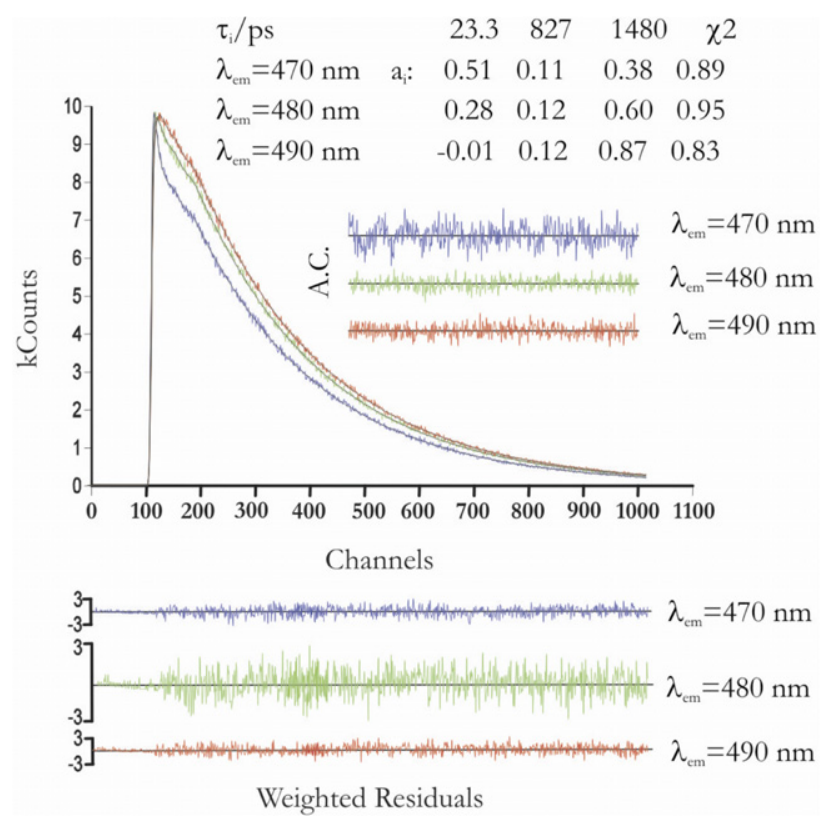

Fig. 4. Fluorescence decays for MPOPV3A in methylcyclohexane, at $293 \mathrm{~K}$, and with excitation at $440 \mathrm{~nm}$, obtained with a TCSPC system with picosecond-time resolution ( $3 \mathrm{ps}$ ). Shown as insets are the decay times and pre-exponential values obtained at three emission wavelengths analysed with global analysis. For a better judgment of the quality of the fits, also shown are the weighted residuals (WR), autocorrelation functions (AC) and chi-square values $\left(\chi^{2}\right)$. exponential decays could properly fit the data (see Fig. 4), via Eq. (3):

$I_{\lambda}(t)=a_{i 1} \mathrm{e}^{-t / \tau_{1}}+a_{i 2} \mathrm{e}^{-t / \tau_{2}}+a_{i 3} \mathrm{e}^{-t / \tau_{3}}$

where the $a_{i j}(j=1,2,3)$ are the pre-exponential factors and $\tau_{i}$ are the decay times with $i=1$ for $\lambda_{\mathrm{em}}=470 \mathrm{~nm}, i=2$ for $\lambda_{\mathrm{em}}=480 \mathrm{~nm}$ and $i=3$ for $\lambda_{\mathrm{em}}=490 \mathrm{~nm}$.

The short decay time appears as a decay-time at the onset $(470 \mathrm{~nm})$ of the emission spectrum and as a rise-time at longer wavelengths $(490 \mathrm{~nm})$. From the reciprocal of this decay time value the rate constant for formation of the relaxed species result was found to be $k=4.3 \times 10^{10} \mathrm{~s}^{-1}$. The pre-exponential coefficient of the middle value decay time $(827 \mathrm{ps})$ is independent of the emission wavelength whereas the longest decay time (1480 ps) changes its contribution with the emission wavelength. In the case of the polyfluorene derivatives [10] previously studied, the fluorescence decays were found to be properly fitted with double exponential laws. However, with polythiophene derivatives only three-exponentials were able to fit the decays [36]. In the present case the middle decay time component cannot be due to a component that does not suffer any structural modification during the lifetime of the other species, i.e., an isolated segment, as observed with the reported study with polythiophenes. In the trimer studied here this component could be due to an impurity and in fact a residual additional peak has been detected by HPLC. However, it may also result from the need of more than one exponential for describing the relaxation process. Thus the unrelaxed trimer predominantly relaxes to a more planar conformation (in ca. $23 \mathrm{ps}$ ), which then decays to the ground state with a lifetime of 1480 ps. Moreover, the relaxed species increases its contribution as the emission wavelength increases which denotes that the relaxed species emission is dominant at longer (lower energy) wavelengths. A complete and detailed time-resolved study (including temperature and emission wavelength dependence) with the overall family of compounds in Scheme 1 together with the MEHPPV polymer is in progress.

\section{Conclusions}

A comprehensive study has been undertaken on the spectral and photophysical properties of six substituted PPV-trimers. The alkyl or alkyloxyl modifications at the peripheral positions of PPV-trimers are shown to induce changes in the photophysical behaviour of the compounds, mostly by increasing the ISC yield with the alkyloxyl derivatives. However, one of the important changes in the photophysical parameters is found when aromatic units of phenylene or anthracene are added to the terminal positions of these compounds. This leads to a decrease of the fluorescence quantum efficiency, particularly in the case of the anthracene substituted compounds. Moreover, one of the most remarkable and important features is the increase in the fluorescence quantum yield upon going to solid state matrices. This is in contrast with the general 
findings on related systems where the fluorescence efficiency is generally known to decrease in the solid state when compared to solution media. Although the method for determining fluorescence quantum yields in films is not the same as used for solutions, and thus the values obtained implicitly possess some uncertainties, it is not likely that more rigorous determination would change the pattern found. As a consequence, the present data strongly suggest that use of these oligomers is a promising pathway for development of synthetic routes for their application in LED devices.

Moreover, we have extended the present study to the excited state conformational behaviour of one of the PPV-trimers. The results are consistent with the occurrence of a fast conformational relaxation of the initially excited oligomer, leading to a more planar conjugation segment. The assignment is unambiguous since the RET mechanism is not possible with the trimers studied, in clear contrast to the general situation found for related polymer systems.

\section{Acknowledgement}

We are indebted to Professor E. Thorn-Csányi and Drs. M. Griepentrog, F. Scheliga (University of Hamburg) for the compounds. Financial support from FEDER and FCT (through projects POCI/QUI/55672/2004 and POCI/QUI/58291/2004) is acknowledged. J.P. acknowledges FCT for a Ph.D. Grant (SFRH/BD/18876/2004) and R.E.D.P. acknowledges Fundação para a Ciência e Tecnologia for a Postdoctoral Grant (BPD/14414/2003). We also thank M. Fernandes for assistance in some of the preliminary photophysical experiments.

\section{References}

[1] R.H. Friend, R.W. Gymer, A.B. Holmes, J.H. Burroughes, R.N. Marks, C. Taliani, D.D.C. Bradley, D.A. Dos Santos, J.L. Bredas, M. Logdlund, W.R. Salaneck, Nature 397 (1999) 121.

[2] A.P. Monkman, H.D. Burrows, M.D. Miguel, I. Hamblett, S. Navaratnam, Chem. Phys. Lett. 307 (1999) 303.

[3] A.P. Monkman, H.D. Burrows, I. Hamblett, S. Navaratnam, U. Scherf, C. Schmitt, Chem. Phys. Lett. 327 (2000) 111.

[4] J. Partee, E.L. Frankevich, B. Uhlhorn, J. Shinar, Y. Ding, T.J. Barton, Phys. Rev. Lett. 82 (1999) 3673.

[5] L.P. Candeias, J. Wildeman, G. Hadziioannou, J.M. Warman, J. Phys. Chem. B 104 (2000) 8366.

[6] L.P. Candeias, G. Padmanaban, S. Ramakrishnan, Chem. Phys. Lett. 349 (2001) 394

[7] J. Seixas de Melo, J. Pina, H.D. Burrows, S. Brocke, O. Herzog, E. Thorn-Csanyi, Chem. Phys. Lett. 388 (2004) 236.

[8] D. Beljonne, Z. Shuai, J. Cornil, J.P. Calbert, J.L. Bredas, J. Photochem. Photobiol. A 144 (2001) 57.
[9] J.L. Bredas, D. Beljonne, V. Coropceanu, J. Cornil, Chem. Rev. 104 (2004) 4971

[10] F.B. Dias, A.L. Maçanita, J. Seixas de Melo, H.D. Burrows, R. Güntner, U. Scherf, A.P. Monkman, J. Chem. Phys. 118 (2003) 7119.

[11] J.H. Hsu, W.S. Fann, H.F. Meng, E.S. Chen, E.C. Chang, S.A. Chen, K.W. To, Chem. Phys. 269 (2001) 367.

[12] R.D. Scurlock, B.J. Wang, P.R. Ogilby, J.R. Sheats, R.L. Clough, J. Am. Chem. Soc. 117 (1995) 10194.

[13] C. Collison, V. Treemaneekarn, W.J. Oldham, J.H. Hsu, L.J. Rothberg, Synth. Met. 119 (2001) 515.

[14] E.J.W. List, J. Partee, J. Shinar, U. Scherf, K. Mullen, E. Zojer, K. Petritsch, G. Leising, W. Graupner, Phys. Rev. B 61 (2000) 10807.

[15] H.D. Burrows, J. Seixas de Melo, C. Serpa, L.G. Arnaut, A.P. Monkman, I. Hamblett, S. Navaratnam, J. Chem. Phys. 115 (2001) 9601.

[16] F.Z. Henari, H. Manaa, K.P. Kretsch, W.J. Blau, H. Rost, S. Pfeiffer, A. Teuschel, H. Tillmann, H.H. Horhold, Chem. Phys. Lett. 307 (1999) 163.

[17] O. Narwark, S.C.J. Meskers, R. Peetz, E. Thorn-Csanyi, H. Bassler, Chem. Phys. 294 (2003) 1.

[18] O. Narwark, A. Gerhard, S.C.J. Meskers, S. Brocke, E. ThornCsanyi, H. Bassler, Chem. Phys. 294 (2003) 17.

[19] J. Seixas de Melo, R.S. Becker, F. Elisei, A.L. Maçanita, J. Chem. Phys. 107 (1997) 6062.

[20] R.S. Becker, J. Seixas de Melo, A.L. Maçanita, F. Elisei, J. Phys. Chem. 100 (1996) 18683.

[21] S. Murov, I. Charmichael, G.L. Hug, Handbook of Photochemistry, M. Dekker Inc., New York, 1993.

[22] I. Carmichael, G.L. Hug, J. Chem. Phys. Ref. Data 15 (1986) 1.

[23] J. Seixas de Melo, L.M. Silva, M. Kuroda, J. Chem. Phys. 115 (2001) 5625.

[24] C.V. Kumar, L. Qin, P.K. Das, J. Chem. Soc., Faraday Trans 280 (1984) 783.

[25] J.C. Lima, I. Abreu, R. Brouillard, A.L. Maçanita, Chem. Phys. Lett. 298 (1998) 189.

[26] G. Striker, in: M. Bouchy (Ed.), Deconvolution and Reconvolution of Analytical Signals, University Press, Nancy, 1982.

[27] J.C. de Mello, H.F. Wittmann, R.H. Friend, Adv. Mater. 9 (1997) 230.

[28] L.O. Palsson, A.P. Monkman, Adv. Mater. 14 (2002) 757.

[29] H.D. Burrows, J. Seixas de Melo, C. Serpa, L.G. Arnaut, M.D.G. Miguel, A.P. Monkman, I. Hamblett, S. Navaratnam, Chem. Phys. 285 (2002) 3 .

[30] Y. Kawashima, T. Hashimoto, H. Nakano, K. Hirao, Theor. Chem. Acc. 102 (1999) 49.

[31] D. Oelkrug, J. Gierschner, H.J. Egelhaaf, L. Luer, A. Tompert, K. Mullen, U. Stalmach, H. Meier, Synth. Met. 121 (2001) 1693.

[32] A.P. Monkman, H.D. Burrows, I. Hamblett, S. Navarathnam, M. Svensson, M.R. Andersson, J. Chem. Phys. 115 (2001) 9046.

[33] B.S. Nehls, F. Galbrecht, A. Bilge, D.J. Brauer, C.W. Lehmann, U. Scherf, T. Farrell, Org. Biomol. Chem. 3 (2005) 3213.

[34] J. Pei, W.L. Yu, W. Huang, A.J. Heeger, Acta Polym. 50 (1999) 327.

[35] S. Westenhoff, C. Daniel, R.H. Friend, C. Silva, V. Sundstrom, A. Yartsev, J. Chem. Phys. 122 (2005).

[36] J. Seixas de Melo, N. Coelho, H.D. Burrows, A. Macanita, F.B. Dias, A.P. Monkman (unpublished results). 